\title{
A Card-type Odor Identification Test for Japanese Patients with Parkinson's Disease and Related Disorders
}

\author{
Yuji Watanabe ${ }^{1}$, Keisuke Suzuki ${ }^{1}$, Tomoyuki Miyamoto ${ }^{2}$, Masayuki Miyamoto ${ }^{3}$, \\ Ayaka Numao ${ }^{1}$, Hiroaki Fujita ${ }^{1}$, Tomoyuki Uchiyama ${ }^{1,4}$, Taro Kadowaki ${ }^{1}$, \\ Takeo Matsubara ${ }^{1}$ and Koichi Hirata ${ }^{1}$
}

\begin{abstract}
:
Objective The characteristics of olfactory impairment in Parkinson's disease (PD), multiple system atrophy (MSA) and progressive supranuclear palsy (PSP) have not been determined in detail. We assessed the olfactory function among PD, MSA and PSP patients.

Methods A card-type odor identification test, Open Essence (OE, Wako, Japan), which consists of 12 different odorants familiar to Japanese subjects, was administered to 98 PD patients, 32 MSA patients, 17 PSP patients and 96 control subjects $\geq 50$ years of age.

Results The PD patients had significantly lower OE scores than the other groups. The OE scores of the MSA and PSP patients fell between those of the PD patients and the control subjects. A cut-off OE score of 6 was beneficial for differentiating PD patients from controls with $84.7 \%$ sensitivity and $85.4 \%$ specificity. A cut-off OE score of 4 had $60.2 \%$ sensitivity and $77.6 \%$ specificity for differentiating PD patients from MSA and PSP patients. The correct answer rates for the curry, Japanese orange and perfume odorants in the PD patients were lower than those in the MSA and PSP patients and controls. The PD patients also had the highest ratio of "not detected" choices across the 12 odors.

Conclusion Marked olfactory impairment was a feature of the patients with PD, while mild olfactory impairment was observed in those with MSA or PSP. The answer patterns and the specific odorants may also be useful in differentiating PD from related disorders.
\end{abstract}

Key words: olfactory function, atypical parkinsonian syndrome, Parkinson's disease, progressive supranuclear palsy, multiple system atrophy, Open Essence

(Intern Med 56: 2871-2878, 2017)

(DOI: 10.2169/internalmedicine.8565-16)

\section{Introduction}

Olfactory impairment is an early non-motor symptom of Parkinson' disease (PD) and is present in a large proportion of PD patients. A number of studies have reported impaired odor detection, identification and discrimination in patients with PD $(1,2)$. During the premotor phase, alpha-synuclein pathology occurs in the olfactory bulb and anterior olfactory nucleus (3). In a three-year-longitudinal study, the presence of severe olfactory impairment at baseline predicted the emergence of dementia and focal changes in brain structures such as the amygdala and other limbic structures (4). In contrast to the marked olfactory impairment that occurs in PD, olfactory impairment has been reported to be mild in atypical parkinsonian syndromes (APSs), such as multiple system atrophy (MSA) and progressive supranuclear palsy (PSP) $(1,5)$. Therefore, a detailed assessment of the olfactory function may be useful in differentiating PD from APSs, such as MSA and PSP, as well as for predicting a

\footnotetext{
${ }^{1}$ Department of Neurology, Dokkyo Medical University, Japan, ${ }^{2}$ Department of Neurology, Dokkyo Medical University Koshigaya Hospital, Japan, ${ }^{3}$ Department of Clinical Medicine for Nursing, Dokkyo Medical University School of Nursing, Japan and ${ }^{4}$ Neuro-urology and Continence Center, Dokkyo Medical University Hospital, Japan

Received: November 15, 2016; Accepted: April 5, 2017; Advance Publication by J-STAGE: September 25, 2017

Correspondence to Dr. Keisuke Suzuki, keisuke@dokkyomed.ac.jp
} 
specific clinical phenotype of PD.

Although the odor stick identification test (OSIT) has consistently revealed significant olfactory impairment in patients with PD compared with those with APSs (6), the characteristics of olfactory impairment in PD and APSs have not been determined in detail. The OSIT for Japanese (OSIT-J) is a useful and widely used tool for olfactory assessment. However, its routine use for clinical practice is sometimes difficult, as it should be refrigerated and requires application, rubbing and handling steps. In contrast, the recently developed Open Essence (OE, Wako, Japan), a selfadministered card-type odor identification test, is quick and easy to perform and can be stored at room temperature $(7,8)$.

In this study, we compared olfactory impairment among patients with PD-related disorders, including PD, MSA and PSP, using OE.

\section{Materials and Methods}

This cross-sectional, case-controlled study was performed at the Department of Neurology, Dokkyo Medical University between January 2012 and May 2016. It was performed in accordance with the Declaration of Helsinki and was approved by the institutional review board of Dokkyo Medical University. Written informed consent was obtained from all subjects who participated in the study.

\section{Subjects}

A total of 147 patients with PD-related disorders (98 PD patients, 32 MSA patients and 17 PSP patients) and 96 controls subjects $\geq 50$ years of age were included in this study. Control subjects were recruited from among a population of stable patients without neurological disorders (dementia disorders, PD-related disorders, multiple sclerosis, myasthenia gravis, polyneuropathies or stroke) who visited our outpatient clinic and from medical staff in our hospital. A diagnosis of PD was made based on the UK PD Society Brain Bank clinical diagnostic criteria (9). MSA and PSP were diagnosed according to established criteria $(10,11)$. Patients with secondary parkinsonism due to medication, vascular lesions or trauma were excluded. Patients with Mini-Mental State Examination (MMSE) scores $<20$ and those with anosmia due to nasal sinus disease, trauma or other causes were excluded based on their history. Among the patients with MSA, 22 had MSA with predominant cerebellar ataxia (MSA-C), and 10 had MSA with predominant parkinsonism (MSA-P). Among the patients with PSP, 15 had Richardson's syndrome, and 2 had PSP-parkinsonism. The disease severity was evaluated based on the Hoehn and Yahr (HY) stage. The cognitive function was evaluated using the MMSE. The levodopa equivalent dose (LED) was calculated in accordance with previously described methods (12).

\section{Olfactory function test}

The card-type odor identification test OE (Wako) was used to assess the olfactory function. OE was developed in Japan to assess olfactory identification and includes 12 different odors familiar to Japanese people: India ink, wood, perfume, menthol, Japanese orange, curry, gas for household use, rose, hinoki cypress, sweaty socks, condensed milk, and roasted garlic. When the subject opens the twice-folded card, the microcapsule breaks, and the odor is released. The subject is asked to choose one of six possible answers: correct odor; odor closest to the correct one; odor close to the correct one; odor very different from the correct one; detectable but not recognizable (unknown); and no smell detected (not detected) $(7,8)$. The $\mathrm{OE}$ test does not require a forced choice from the subjects. The OE test also consists of odorants compatible with the OSIT for Japanese and can be performed more easily and faster than the OSIT-J because it skips the application, rubbing and handling steps required for that test $(7,8)$.

The reliability of the $\mathrm{OE}$ test has been confirmed by its significant and strong correlation with the average detection and recognition thresholds of Jet Stream Olfactometry, symptom scores, and duration scores for the intravenous olfactory test (7). A previous study determined that an $\mathrm{OE}$ score $\leq 7$ was abnormal and that this cut-off was useful in differentiating subjects with olfactory dysfunction (mean age 57 years) from healthy volunteers (mean age 35 years) (7); however, because appropriate OE score cut-off values for differentiating among PD-related disorders have not been evaluated, we determined the optimum OE cut-off scores as well as the number of "not determined" choices for OE subitems for each participant to discriminate PD from MSA and PSP based on a receiver operating characteristic (ROC) curve analysis. The OE total and sub-scores in each group were calculated and correlated with clinical factors.

\section{Statistical analyses}

A one-way analysis of variance followed by Bonferroni test was used to compare continuous variables among the groups. A general linear model was applied to compare the OE total score among the groups, controlling for age and sex. The chi-squared or Fisher's exact test was used to compare categorical variables. The ROC curve was utilized to determine optimum OE score cut-off points to differentiate PD from APS or controls. Spearman's rank correlation coefficients were used for correlation analyses. Statistical significance was defined as a two-tailed $\mathrm{p}<0.05$. The GraphPad Prism for Windows software program (Version 5.01; GraphPad Software, San Diego, USA) was used to generate figures, and the IBM SPSS Statics 22.0 software program (IBM SPSS, Tokyo, Japan) was used for the statistical analysis.

\section{Results}

The clinical characteristics of the patients with PD, MSA and PSP and of the controls are shown in Table 1. The PD patients had a longer disease duration and higher LED than 
Table 1. Clinical Characteristics of the Patients with PD, MSA, and PSP as Well as the Controls.

\begin{tabular}{lccccc}
\hline & PD $(\mathrm{n}=98)$ & MSA $(\mathrm{n}=32)$ & PSP $(\mathrm{n}=17)$ & Controls $(\mathrm{n}=96)$ & $\mathrm{p}$ value \\
\hline Male/female & $46 / 52$ & $15 / 17$ & $7 / 10$ & $43 / 53$ & 0.97 \\
Age (years) & $68.9 \pm 8.0(51-88)$ & $67.2 \pm 7.8(51-82)$ & $71.6 \pm 7.9(50-82)$ & $66.3 \pm 7.7(50-82)$ & 0.069 \\
Smoking status & & & & 0.077 \\
$\quad$ Never, $\mathrm{n}(\%)$ & $80(81.6)$ & $23(71.9)$ & $14(82.4)$ & $61(63.5)$ & \\
Past, $\mathrm{n}(\%)$ & $15(15.3)$ & $6(18.8)$ & $1(5.9)$ & $26(27.1)$ & \\
Current, n $(\%)$ & $3(3.1)$ & $3(9.4)$ & $2(11.8)$ & $9(9.4)$ & 0.10 \\
MMSE & $27.1 \pm 2.8$ & $26.0 \pm 2.8$ & $26.1 \pm 2.8$ & - & 0.019 \\
Disease duration (years) & $4.4 \pm 4.6$ & $2.6 \pm 2.0$ & $2.2 \pm 1.5$ & - & 0.083 \\
HY stage & $2.7 \pm 0.9$ & $3.1 \pm 1.0$ & $3.0 \pm 0.6$ & - & 0.098 \\
Drug naïve patients, n (\%) & $56(57.1)$ & $25(78.1)$ & $11(64.7)$ & - & 0.0028 \\
LED (mg/day) & $304.7 \pm 404.9$ & $64.5 \pm 145.0$ & $144.5 \pm 216.2$ & - & - \\
\hline
\end{tabular}

PD: Parkinson's disease, MSA: multiple system atrophy, PSP: progressive supranuclear palsy, HY: Hoehn and Yahr, LED: levodopa equivalent dose

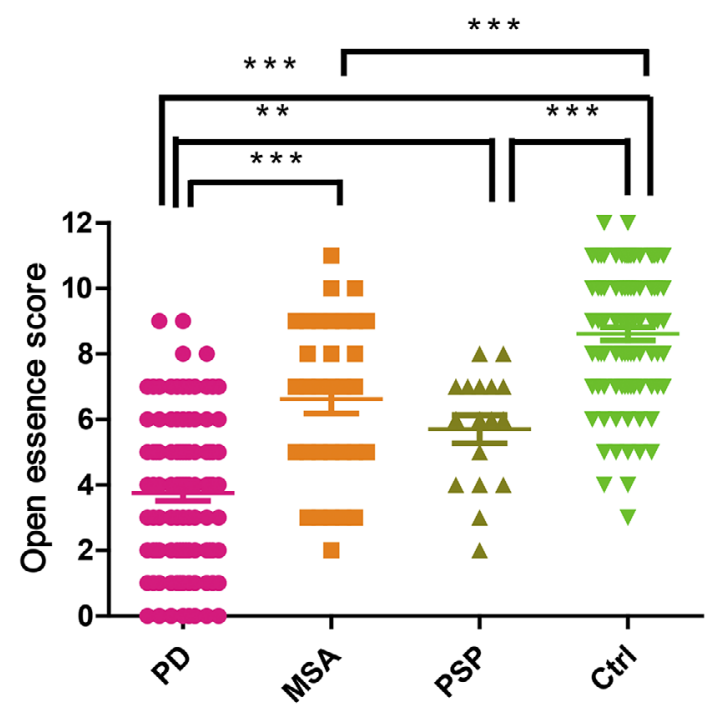

Figure 1. Comparison of the $\mathrm{OE}$ total scores among the patients with PD, MSA and PSP and controls. *p<0.05, **p<0.01, $* * * \mathbf{p}<0.001, * * * * \mathbf{p}<0.0001$. PD: Parkinson's disease, MSA: multiple system atrophy, PSP: progressive supranuclear palsy, Ctrl: controls, OE: Open Essence

the MSA and PSP patients. The OE scores of the controls $(8.6 \pm 2.0)$ were significantly higher than those of the PD $(3.8$ $\pm 2.3)$, MSA (6.6 \pm 2.5$)$ and PSP patients $(5.7 \pm 1.8)(\mathrm{p}<0.0001$, Fig. 1). This difference remained significant after adjusting for sex and age (PD, 3.8 \pm 2.2 ; MSA, 6.6 \pm 2.2 ; PSP, 5.8 \pm 2.2 ; controls, 8.5 $\pm 2.1 ; \mathrm{p}<0.0001)$. Among the patients with parkinsonism, the PD patients had significantly lower OE scores than the MSA and PSP patients. The OE scores of the MSA and PSP patients fell between those of the PD patients and control subjects. There was no significant difference in the OE scores between MSA-C $(6.5 \pm 2.5)$ and MSAP patients $(7.0 \pm 2.6)(\mathrm{p}=0.58)$.

According to ROC curve analysis, OE score cut-offs of 4, 5 and 6 had $60.2 \%$ sensitivity and $77.6 \%$ specificity, $75.5 \%$ sensitivity and $61.2 \%$ specificity and $84.7 \%$ sensitivity and $53.1 \%$ specificity, respectively, for differentiating PD from
APS (area under the curve, 0.77; p<0.0001) (Fig. 2). An OE score cut-off of 7 had high sensitivity (95.9\%) but low specificity (30.6\%) in differentiating PD from APS. In contrast, in differentiating PD from controls, OE cut-offs of 5, 6 and 7 yielded $75.5 \%$ sensitivity and $91.7 \%$ specificity, $84.7 \%$ sensitivity and $85.4 \%$ specificity and $95.9 \%$ sensitivity and $71.9 \%$ specificity, respectively (area under the curve, 0.94; p<0.0001) (Fig. 2).

When an OE score cut-off of 4 was used to define olfactory impairment, the percentage of individuals with olfactory impairment among the patients with PD-related disorders and the controls was as follows: PD, $60.2 \%$; MSA, $18.8 \%$; PSP, $29.4 \%$ and controls, $3.1 \%$. The PD patients with olfactory impairment (OE score $\leq 4)$ had a lower MMSE score than those without olfactory impairment ( $\mathrm{OE}$ score $>4)$ (Table 2). The MSA patients with olfactory impairment had lower MMSE scores and LEDs than those without. One $(10.0 \%)$ patient with MSA-P and $5(22.7 \%)$ patients with MSA-C showed an OE score $\leq 4(\mathrm{p}=0.64)$. The PSP patients with olfactory impairment were older than those without olfactory impairment. Among the PD-related disorders, olfactory impairment was not related to disease duration, HY stage or smoking status.

The OE scores correlated negatively with age in the patients with PD and PSP as well as in the controls. A positive correlation between MMSE and OE scores was found in the PD and MSA patients, and this correlation was stronger in the MSA patients $(r=0.49)$ than in the PD patients $(r=0.32)$ (Fig. 3A and B). We therefore analyzed the correlation of MMSE sub-items, such as orientation for time and place, registration/recall and calculation/attention (serial seven subtraction), with OE scores among PD, MSA and PSP patients. In PD and PSP patients, there was no significant correlation of OE with MMSE sub-items. In contrast, in MSA patients, although there was no significant correlation of $\mathrm{OE}$ scores with registration/recall or orientation, a positive correlation was found between calculation/attention and $\mathrm{OE}$ scores $(r=0.64, p<0.01)$.

With regard to the OE sub-items, the PD patients had sig- 


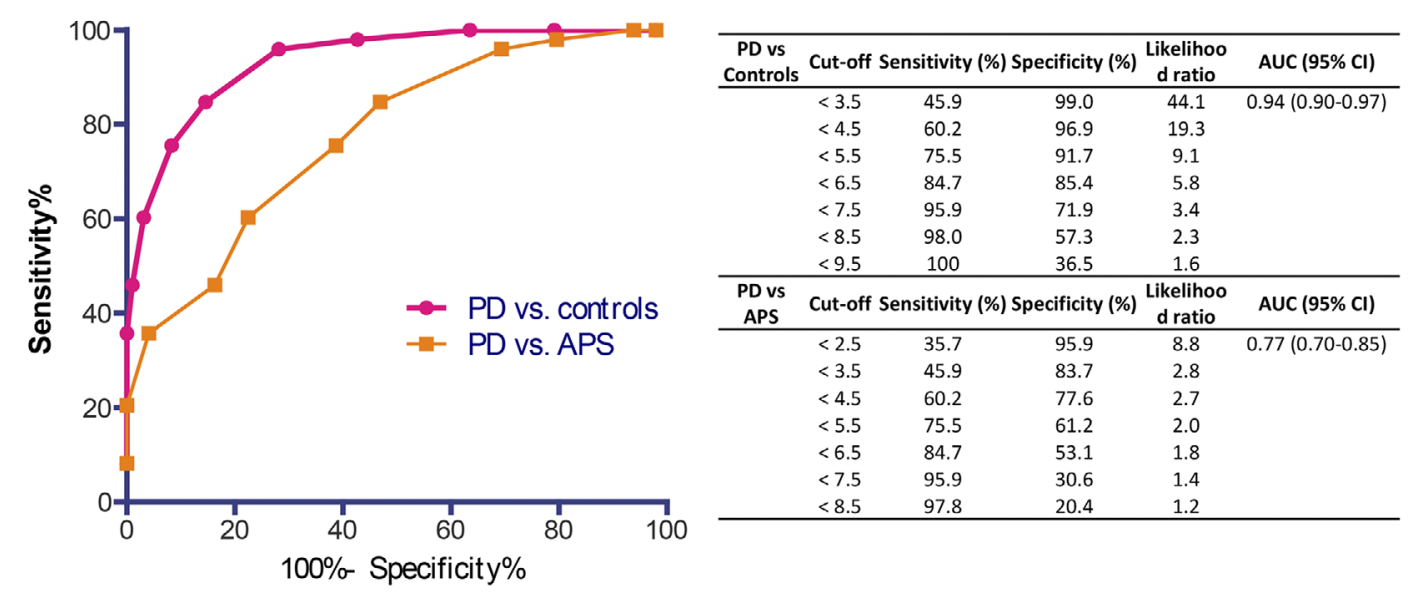

Figure 2. ROC curve of Open Essence. The ROC curves of Open Essence for differentiating PD from controls and PD from APS are shown. PD: Parkinson's disease, APS: atypical parkinsonian syndrome

Table 2. Clinical Characteristics of the Patients with and without Olfactory Impairment.

\begin{tabular}{lcccccc}
\hline & \multicolumn{2}{c}{ PD $(\mathrm{n}=98)$} & \multicolumn{2}{c}{ MSA $(\mathrm{n}=32)$} & \multicolumn{2}{c}{ PSP $(\mathrm{n}=17)$} \\
\hline & $\mathrm{OE} \leq 4$ & $\mathrm{OE}>4$ & $\mathrm{OE} \leq 4$ & $\mathrm{OE}>4$ & $\mathrm{OE} \leq 4$ & OE $>4$ \\
$\mathrm{~N}$ & 59 & 39 & 6 & 26 & 5 & 12 \\
Male/female & $32 / 27$ & $14 / 25$ & $5 / 1$ & $10 / 16$ & $1 / 4$ & $6 / 6$ \\
Age (years) & $69.8 \pm 8.3$ & $67.6 \pm 7.5$ & $72.7 \pm 8.7$ & $66.0 \pm 7.2$ & $77.4 \pm 3.6 *$ & $69.2 \pm 7.9$ \\
Smoking status & & & & & & \\
$\quad$ Never, n (\%) & $47(79.7)$ & $33(84.6)$ & $5(83.3)$ & $18(69.2)$ & $5(100.0)$ & $9(75.0)$ \\
$\quad$ Past, n (\%) & $9(15.3)$ & $6(15.4)$ & $1(16.7)$ & $5(19.2)$ & $0(0.0)$ & $1(8.3)$ \\
$\quad$ Current, n (\%) & $3(5.1)$ & $0(0.0)$ & $0(0.0)$ & $3(11.5)$ & $0(0.0)$ & $2(16.7)$ \\
MMSE & $26.2 \pm 2.9 * * *$ & $28.5 \pm 2.2$ & $23.5 \pm 1.4 * *$ & $26.6 \pm 2.6$ & $25.2 \pm 2.3$ & $26.5 \pm 3.0$ \\
Disease duration (years) & $4.3 \pm 4.0$ & $4.6 \pm 5.4$ & $2.3 \pm 2.0$ & $2.6 \pm 2.0$ & $2.8 \pm 1.5$ & $2.0 \pm 1.5$ \\
HY stage & $2.8 \pm 0.8$ & $2.5 \pm 0.9$ & $3.5 \pm 1.0$ & $3.0 \pm 0.9$ & $2.7 \pm 0.4$ & $3.1 \pm 0.7$ \\
Drug-naïve patients, $\mathrm{n}(\%)$ & $31(52.5)$ & $25(64.1)$ & $5(83.3)$ & $20(76.9)$ & $3(60.0)$ & $8(66.7)$ \\
LED (mg/day) & $324.4 \pm 389.5$ & $275.5 \pm 430.1$ & $0.0 \pm 0.0 *$ & $80.0 \pm 158.1$ & $162.5 \pm 261.3$ & $136.4 \pm 206.3$ \\
\hline
\end{tabular}

$\mathrm{OE} \leq 4$ vs. OE $>4,{ }^{*} \mathrm{p}<0.05,{ }^{*} \mathrm{p}<0.01, * * * \mathrm{p}<0.001$

PD: Parkinson's disease, MSA: multiple system atrophy, PSP: progressive supranuclear palsy, HY: Hoehn and Yahr, LED: levodopa equivalent dose, OE: Open Essence

nificantly lower correct answer rates for all 12 odors than the controls. The correct answer rates for perfume, Japanese orange and curry were significantly lower in PD patients than in PD, MSA and PSP patients and controls (Fig. 4, Table 3). Compared with the MSA patients, the PD patients had lower correct answer rates for perfume, Japanese orange, curry, condensed milk, sweaty-smelling socks, India ink and Japanese hinoki cypress. Furthermore, the answer patterns for the 12 odorants differed between the patients with PD-related disorders and the controls (Fig. 4). The combined percentage of responses of "unknown" and "not detected" across all of the odors was lowest in the controls, while the percentage of "not detected" responses for all 12 odors was highest in the PD patients. In differentiating PD from MSA and PSP, 2 or more responses of "not detected" or "unknown" for the OE sub-items, irrespective of the type of odor, yielded $61.2 \%$ sensitivity and $61.2 \%$ specificity, whereas 3 or more responses of "not detected" or "unknown" for the OE sub-items had $43.9 \%$ sensitivity and
$73.5 \%$ specificity (area under the curve, $0.63 ; \mathrm{p}=0.0085$ ).

\section{Discussion}

In this study, we found that PD patients had significantly lower total scores of OE than PSP and MSA patients and control subjects. Because olfaction is influenced by age (13), we included the patients and controls $\geq 50$ years of age. This finding is in agreement with the marked olfactory impairment that has been reported in PD patients across studies despite the use of different methodologies to assess odor threshold, identification and discrimination (1). Furthermore, by using $\mathrm{OE}$, our study confirmed the previously reported usefulness of olfactory testing in differentiating PD from MSA and PSP patients (14-16). An ROC curve analysis showed that an OE score cut-off of 4 was useful for differentiating PD from MSA and PSP (60.2\% sensitivity and $77.6 \%$ specificity), whereas a cut-off score of 6 was beneficial for differentiating PD patients from controls with $84.7 \%$ 

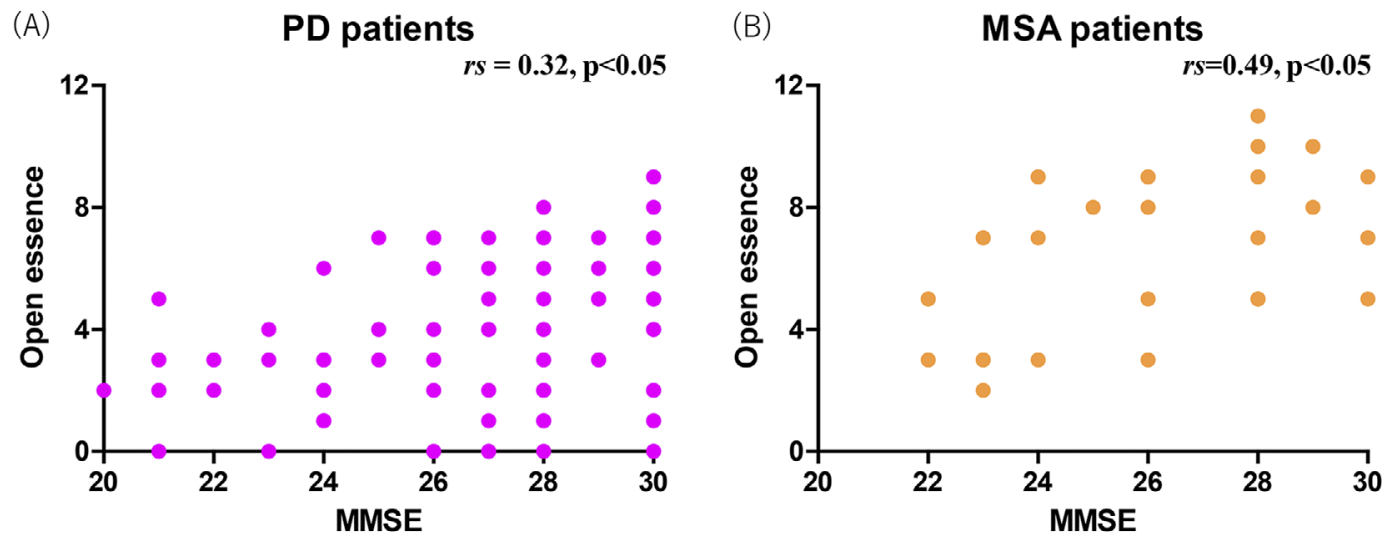

Figure 3. Correlation between the OE and MMSE score in PD and MSA patients. (A) PD patients, (B) MSA patients. rs: Spearman's rank correlation coefficient, PD: Parkinson's disease, MSA: multiple system atrophy

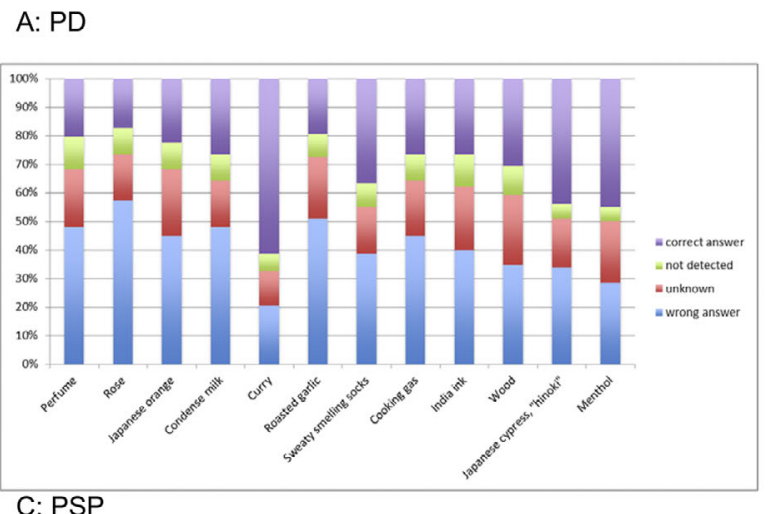

B: MSA
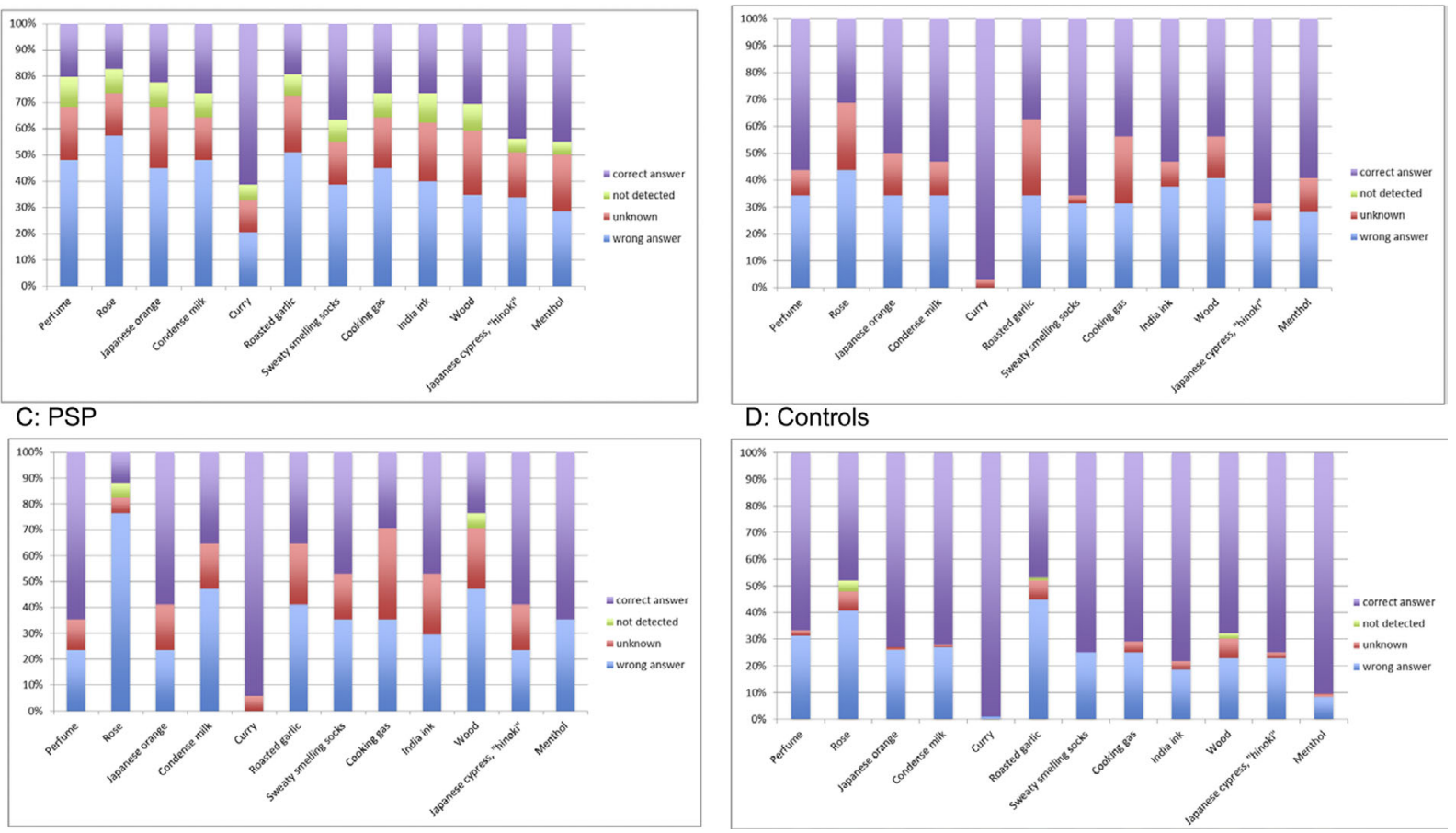

Figure 4. A comparison of the answers for the OE sub-items among the patients with PD, MSA and PSP and controls. PD: Parkinson's disease, MSA: multiple system atrophy, PSP: progressive supranuclear palsy, OE: Open Essence

sensitivity and $85.4 \%$ specificity.

Our study did not reveal a significant correlation between OE score and disease duration in PD patients. Olfactory impairment occurs early and may even appear before the motor symptoms of PD, but several studies have failed to show a correlation of olfactory impairment with disease duration or dopaminergic treatment $(1,17,18)$. Although we did not correlate the $\mathrm{OE}$ scores with the cardiac sympathetic function in this study, olfactory impairment correlates with cardiac sympathetic denervation (19-21). Furthermore, olfactory dysfunction may be an indicator of Lewy body pathology rather than striatal dopamine deficiency $(18,22,23)$.

The answer patterns (correct answer, wrong answer, un- known and not detected) in Fig. 4 revealed that for all of the odors, the rate of response of "not detected", which corresponds to anosmia, was highest in the PD patients compared with the other groups. A further analysis demonstrated that, regardless of odor type, 3 or more "not detected" or "unknown" answers had $43.9 \%$ sensitivity and $73.5 \%$ specificity in discriminating PD from MSA and PSP. Our findings support the hypothesis that bilateral and robust olfactory impairment is a feature of PD (1).

Selective olfactory impairment has also been described in PD patients. Bohnen et al. (24) reported that the impaired identification of 3 specific odors (banana, licorice and dill pickle) had an accuracy of $>75 \%$ for the diagnosis of PD. 
Table 3. Correct Answer Rates for OE Subitems among the Patients with PD, MSA, and PSP as Well as the Controls.

\begin{tabular}{|c|c|c|c|c|c|c|c|c|c|c|c|}
\hline & & & & & $\mathrm{Ctrl}$ & $\begin{array}{l}\text { PD vs. } \\
\text { MSA }\end{array}$ & $\begin{array}{l}\text { PD vs. } \\
\text { PSP }\end{array}$ & $\begin{array}{l}\text { MSA vs. } \\
\text { PSP }\end{array}$ & $\begin{array}{l}\text { PD vs. } \\
\text { Ctrl }\end{array}$ & $\begin{array}{c}\text { MSA } \\
\text { vs. Ctrl }\end{array}$ & $\begin{array}{l}\text { PSP vs. } \\
\text { Ctrl }\end{array}$ \\
\hline & & $(\%)$ & $(\%)$ & $(\%)$ & $(\%)$ & \multicolumn{6}{|c|}{$\mathrm{p}$ value } \\
\hline \multirow{4}{*}{ Sweet odor } & Perfume & 20.0 & 56.3 & 64.7 & 66.7 & $<0.001$ & $<0.001$ & 0.76 & $<0.001$ & 0.30 & 1.0 \\
\hline & Rose & 17.3 & 31.3 & 11.8 & 47.9 & 0.13 & 0.73 & 0.17 & $<0.001$ & 0.15 & 0.0067 \\
\hline & Japanese orange & 22.4 & 50.0 & 58.8 & 72.9 & 0.0064 & 0.0060 & 0.76 & $<0.001$ & 0.029 & 0.26 \\
\hline & Condense milk & 26.5 & 53.1 & 35.3 & 71.9 & 0.0088 & 0.56 & 0.37 & $<0.001$ & 0.081 & 0.0052 \\
\hline \multirow{2}{*}{ Spices } & Curry & 61.2 & 96.9 & 94.1 & 99.0 & $<0.001$ & 0.010 & $>0.99$ & $<0.001$ & 0.15 & 0.28 \\
\hline & Roasted garlic & 19.4 & 37.5 & 35.3 & 46.9 & 0.055 & 0.20 & $>0.99$ & $<0.001$ & 0.41 & 0.44 \\
\hline $\begin{array}{l}\text { Rotten, } \\
\text { excreta }\end{array}$ & $\begin{array}{l}\text { Sweaty smelling } \\
\text { socks }\end{array}$ & 36.7 & 65.6 & 47.1 & 75.0 & 0.0071 & 0.43 & 0.24 & $<0.001$ & 0.36 & 0.039 \\
\hline Gas, smoke & Cooking gas & 26.5 & 43.8 & 29.4 & 70.8 & 0.080 & 0.77 & 0.37 & $<0.001$ & 0.0099 & 0.0018 \\
\hline \multirow{4}{*}{$\begin{array}{l}\text { Wood, } \\
\text { grass, herb }\end{array}$} & India ink & 26.5 & 53.1 & 47.1 & 78.1 & 0.0047 & 0.15 & 0.77 & $<0.001$ & 0.012 & 0.015 \\
\hline & Wood & 30.6 & 43.8 & 23.5 & 67.7 & 0.20 & 0.77 & 0.22 & $<0.001$ & 0.021 & $<0.001$ \\
\hline & $\begin{array}{l}\text { Japanese cypress } \\
\text { hinoki }\end{array}$ & 43.9 & 68.8 & 58.8 & 75.0 & 0.024 & 0.30 & 0.54 & $<0.001$ & 0.50 & 0.24 \\
\hline & Menthol & 44.9 & 59.4 & 64.7 & 90.6 & 0.22 & 0.19 & 0.77 & $<0.001$ & $<0.001$ & 0.011 \\
\hline
\end{tabular}

Significant differences are highlighted in bold.

Hawkes et al. (25) reported that among the 40 odors of the University of Pennsylvania Smell Identification test, failure to identify both pizza and wintergreen was best able to discriminate PD patients from controls. Homma et al. (26) used the OE test to study the olfactory function of Japanese patients with PD and other neurological diseases and healthy controls and showed that the identification rate for condensed milk and roasted garlic was significantly reduced in PD patients compared with other groups, whereas the identification rates for menthol, cypress wood and wood were similar among the groups. In our study, the identification of condensed milk and roasted garlic was significantly impaired in the PD, MSA and PSP patients compared with the controls, but this distinction could not be used to differentiate the patients with PD-related disorders from the healthy controls. However, we did find that the correct answer rates for three odors (perfume, Japanese orange and curry) were lower in the PD group than in the MSA and PSP groups. Curry odor is intense and familiar to Japanese subjects, but it also stimulates the chemosensory system through the trigeminal nerve (27). This additional activation may explain why the correct answer rate for this odor was higher than that for other odors among the participants in our study. Nevertheless, $39 \%$ of the PD patients were unable to identify the curry odor correctly, indicating the involvement of the central olfactory tract or the chemosensory system in PD.

Our study showed that the correct answer rates for the curry, Japanese orange and perfume odorants in the PD patients were lower than those in the patients with MSA and PSP as well as in the controls. However, in a study of 243 healthy Japanese subjects, $100 \%$ and $99 \%$ correctly identified curry and menthol, respectively, using the OE test. In addition, the combination of scores for cypress wood and India ink odorants was useful for detecting olfactory impairment (OE score 57 ) (28). This study suggested that intense odorants, such as curry and menthol, were not useful for detecting mild olfactory impairment in subjects without neurological disorders, but we showed that the assessment of curry, Japanese orange and perfume odorants was useful for distinguishing PD patients from MSA and PSP patients as well as controls.

In PD, the olfactory bulb and anterior olfactory nucleus, along with the dorsal motor nucleus of the vagus, are the site of induction of Lewy pathology (3); this fact may explain the early occurrence of olfactory impairment in PD. In pathology-based studies, the neuronal loss of the anterior olfactory nucleus correlated with disease duration (5). Tau pathology was detected in the olfactory bulb of cases of pathologically diagnosed Lewy body disease $(5,29)$. In addition, the considerable alpha-synuclein pathology observed in the amygdala (30), piriform cortex (1) and hippocampus (17) in patients with PD may be involved in the olfactory impairment. Damage to largely nondopaminergic neurotransmitter systems (the serotoninergic raphe nucleus, noradrenergic locus ceruleus and cholinergic nucleus basalis of Meynert) may also contribute to olfactory impairment in PD (1). Mrochen et al. (31) demonstrated impairment of the hedonic olfactory range in patients with $\mathrm{PD}$, suggesting the potential involvement of the limbic system. In contrast to the degeneration of the mesocortical and mesolimbic dopaminergic systems, an increase in dopaminergic neurons in the olfactory bulb has been reported in PD (32). This increase may be a compensatory response to substantia nigra degeneration and may explain the levodopaunresponsiveness of the olfactory deficit found in PD (5).

In our study, the PSP and MSA patients exhibited OE scores between those of the PD patients and control subjects, indicating mild hyposmia. Patients with MSA have been reported to show mild impairment of the olfactory function $(15,33)$. In MSA, the appearance of glial cytoplasmatic inclusions in the olfactory bulb, neuronal loss in the 
anterior olfactory nucleus (34) and cerebellar atrophy, which may influence cerebellar involvement in olfactory processing (33), may contribute to mild olfactory impairment. We observed a stronger correlation between olfactory impairment and cognitive impairment in the MSA patients than in the PD patients. Cognitive impairment, particularly frontal executive dysfunction, is now considered an integral part of MSA (35). We also detected a positive correlation between the MMSE sub-items of serial seven subtraction (calculation/attention) in MSA patients only, suggesting that impaired attentional set shifting in MSA (35) may contribute to the impaired olfaction in these patients. Furthermore, while severe olfactory impairment predicts the future development of dementia in PD patients (4), this link has yet to be tested in MSA patients. Whether or not olfactory impairment can predict cognitive impairment as a characteristic of a clinical subtype of MSA requires further study.

In contrast to the findings in PD and MSA patients, PSP patients showed a normal or minimally impaired olfactory function $(5,14-16)$; however, olfactory impairment can progress in parallel with the disease (36). Tau pathology was observed in the olfactory bulb in patients with hyposmia and synucleinopathies, such as PD and dementia with Lewy bodies, but interestingly, in patients with tauopathies, such as PSP or corticobasal degeneration, the olfactory bulb did not show tau pathology $(29,36)$. Therefore, the mild olfactory impairment that was observed in the PSP patients in our study may have been due to the involvement of more central olfactory-related brain structures, such as the orbitofrontal cortex, which is known to be involved in PSP.

One of the limitations of this study is that although we excluded cases of anosmia due to nasal sinus disease from the study, an otorhinolaryngologic examination was not performed at the time of the study. Genetic factors such as the significant loss of smell that occurs after 65 years age more often in men more than women, exposure to smoking and urban living conditions can influence the results of an olfactory assessment (5), and these factors may have influenced the results of our study. The correct answer rates for several odorants, such as perfume (27), rose and roasted garlic (28), were not high in the controls, a result that may be related to the sensitivity of the test. Furthermore, some subjects with hyposmia as a premotor sign of PD may have been included in the controls. Apathy in PD patients has been associated with poor smell identification (37); however, in our study, psychiatric symptoms such as apathy or depression were not assessed. The OE total scores in our controls were lower than those in Japanese healthy controls in previous studies $(7,26)$. This is probably due to differences in inclusion criteria and sample sizes among studies; for controls in our study ( $n=96$ ), we excluded those with anosmia due to known conditions but might have inadvertently included those with mild hyposmia, whereas the studies by Homma (26) and Fujio et al. (7) used healthy controls ( $n=$ 10 and 50, respectively) without any signs of olfactory disturbance or any complaints of olfactory impairment. Never- theless, given that up to one third of elderly subjects can have olfactory impairment due to various reasons, applying a cut-off value of 6 would still be useful for discriminating PD patients from subjects with non-neurological disorders who may have mild olfactory impairment in a clinical setting. Finally, it could be argued that olfactory identification tests assess selective olfactory impairment because odorants activate multiple receptors and are composed of many chemicals; therefore, olfactory impairment may not be related to specific odorants (1).

In conclusion, we found that marked olfactory impairment was a more prominent feature of patients with PD than of those with MSA or PSP, who showed mild olfactory impairment. An evaluation based not only on the OE total score but also on the choices for OE sub-items may be useful for differentiating PD from APSs such as MSA and PSP.

\section{The authors state that they have no Conflict of Interest (COI).}

\section{Acknowledgement}

We thank all of the participants and medical staff for their assistance with this study.

\section{References}

1. Doty RL. Olfaction in Parkinson's disease and related disorders. Neurobiol Dis 46: 527-552, 2012.

2. Haehner A, Hummel T, Reichmann H. A clinical approach towards smell loss in Parkinson's disease. J Parkinsons Dis 4: 189-195, 2014.

3. Braak H, Del Tredici K, Rub U, de Vos RA, Jansen Steur EN, Braak E. Staging of brain pathology related to sporadic Parkinson's disease. Neurobiol Aging 24: 197-211, 2003.

4. Baba T, Kikuchi A, Hirayama K, et al. Severe olfactory dysfunction is a prodromal symptom of dementia associated with Parkinson's disease: a 3 year longitudinal study. Brain 135: 161-169, 2012.

5. Puschmann A, Wszolek ZK. Olfactory dysfunction. In: Parkinson's Disease and Nonmotor Dysfunction. 2nd ed. Pfeiffer RF, BodisWollner I, Eds. Humana Press, New York, 2013: 335-348.

6. Kikuchi A, Baba T, Hasegawa T, Sugeno N, Konno M, Takeda A. Differentiating Parkinson's disease from multiple system atrophy by [123I] meta-iodobenzylguanidine myocardial scintigraphy and olfactory test. Parkinsonism Relat Disord 17: 698-700, 2011.

7. Fujio H, Doi K, Hasegawa S, Kobayakawa T, Nibu K. Evaluation of card-type odor identification test for Japanese patients with olfactory disturbance. Ann Otol Rhinol Laryngol 121: 413-418, 2012.

8. Saito S, Ayabe S, Naito N. Development of an odor identification test for Japanese people: verification of stick type and card type. J Jpn Assoc Odor Environ 34: 1-6, 2003.

9. Hughes AJ, Daniel SE, Kilford L, Lees AJ. Accuracy of clinical diagnosis of idiopathic Parkinson's disease: a clinico-pathological study of 100 cases. J Neurol Neurosurg Psychiatry 55: 181-184, 1992.

10. Gilman S, Wenning GK, Low PA, et al. Second consensus statement on the diagnosis of multiple system atrophy. Neurology $\mathbf{7 1}$ : 670-676, 2008.

11. Litvan I, Agid Y, Calne D, et al. Clinical research criteria for the diagnosis of progressive supranuclear palsy (Steele-RichardsonOlszewski syndrome): report of the NINDS-SPSP international workshop. Neurology 47: 1-9, 1996.

12. Tomlinson CL, Stowe R, Patel S, Rick C, Gray R, Clarke CE. 
Systematic review of levodopa dose equivalency reporting in Parkinson's disease. Mov Disord 25: 2649-2653, 2010.

13. Doty RL, Kamath V. The influences of age on olfaction: a review. Front Psychol 5: 20, 2014.

14. Doty RL, Golbe LI, McKeown DA, Stern MB, Lehrach CM, Crawford D. Olfactory testing differentiates between progressive supranuclear palsy and idiopathic Parkinson's disease. Neurology 43: 962-965, 1993.

15. Wenning GK, Shephard B, Hawkes C, Petruckevitch A, Lees A, Quinn N. Olfactory function in atypical parkinsonian syndromes. Acta Neurol Scand 91: 247-250, 1995.

16. Suzuki M, Hashimoto M, Yoshioka M, Murakami M, Kawasaki K, Urashima M. The odor stick identification test for Japanese differentiates Parkinson's disease from multiple system atrophy and progressive supra nuclear palsy. BMC Neurol 11: 157, 2011.

17. Hawkes C. Olfaction in neurodegenerative disorder. Mov Disord 18: 364-372, 2003.

18. Goldstein DS, Sewell L. Olfactory dysfunction in pure autonomic failure: implications for the pathogenesis of Lewy body diseases. Parkinsonism Relat Disord 15: 516-520, 2009.

19. Lee PH, Yeo SH, Yong SW, Kim YJ. Odour identification test and its relation to cardiac 123I-metaiodobenzylguanidine in patients with drug induced parkinsonism. J Neurol Neurosurg Psychiatry 78: 1250-1252, 2007.

20. Hattori T, Orimo S, Hallett M, et al. Relationship and factor structure in multisystem neurodegeneration in Parkinson's disease. Acta Neurol Scand 130: 347-353, 2014.

21. Oka H, Toyoda C, Yogo M, Mochio S. Olfactory dysfunction and cardiovascular dysautonomia in Parkinson's disease. J Neurol 257: 969-976, 2010.

22. Driver-Dunckley E, Adler CH, Hentz JG, et al. Olfactory dysfunction in incidental Lewy body disease and Parkinson's disease. Parkinsonism Relat Disord 20: 1260-1262, 2014.

23. Miyamoto T, Miyamoto M, Iwanami M, Suzuki K, Inoue $Y$, Hirata $\mathrm{K}$. Odor identification test as an indicator of idiopathic REM sleep behavior disorder. Mov Disord 24: 268-273, 2009.

24. Bohnen NI, Gedela S, Kuwabara H, et al. Selective hyposmia and nigrostriatal dopaminergic denervation in Parkinson's disease. J Neurol 254: 84-90, 2007.

25. Hawkes CH, Shephard BC. Selective anosmia in Parkinson's disease? Lancet 341: 435-436, 1993.

26. Homma H, Yoritaka A, Hattori N, Kobayakawa T, Ikeda K. Clini- cal application of a card-type odor identification test to olfactory assessment in Parkinson's disease. Auris Nasus Larynx 40: 173176, 2013.

27. Okutani F, Hirose K, Kobayashi T, Kaba H, Hyodo M. Evaluation of "Open Essence" odor-identification test card by application to healthy volunteers. Auris Nasus Larynx 40: 76-80, 2013.

28. Shiga H, Yamamoto J, Kitamura M, et al. Combinations of two odorants of smell identification test for screening of olfactory impairment. Auris Nasus Larynx 41: 523-527, 2014.

29. Tsuboi Y, Wszolek ZK, Graff-Radford NR, Cookson N, Dickson DW. Tau pathology in the olfactory bulb correlates with Braak stage, Lewy body pathology and apolipoprotein epsilon4. Neuropathol Appl Neurobiol 29: 503-510, 2003.

30. Harding AJ, Stimson E, Henderson JM, Halliday GM. Clinical correlates of selective pathology in the amygdala of patients with Parkinson's disease. Brain 125: 2431-2445, 2002.

31. Mrochen A, Marxreiter F, Kohl Z, et al. From sweet to sweat: hedonic olfactory range is impaired in Parkinson's disease. Parkinsonism Relat Disord 22: 9-14, 2016.

32. Huisman E, Uylings HB, Hoogland PV. A $100 \%$ increase of dopaminergic cells in the olfactory bulb may explain hyposmia in Parkinson's disease. Mov Disord 19: 687-692, 2004.

33. Abele M, Riet A, Hummel T, Klockgether T, Wullner U. Olfactory dysfunction in cerebellar ataxia and multiple system atrophy. J Neurol 250: 1453-1455, 2003.

34. Kovacs T, Papp MI, Cairns NJ, Khan MN, Lantos PL. Olfactory bulb in multiple system atrophy. Mov Disord 18: 938-942, 2003.

35. Stankovic I, Krismer F, Jesic A, et al. Cognitive impairment in multiple system atrophy: a position statement by the Neuropsychology Task Force of the MDS Multiple System Atrophy (MODIMSA) study group. Mov Disord 29: 857-867, 2014.

36. Reichmann H, Herting B. Olfaction in Parkinson's disease. In: Non-Motor Symptoms of Parkinson's Disease. Chaudhuri KR, Tolosa E, Schapira A, Poewe W, Eds. OUP Oxford, New York, 2009: 279-285.

37. Cramer CK, Friedman JH, Amick MM. Olfaction and apathy in Parkinson's disease. Parkinsonism Relat Disord 16: 124-126, 2010.

The Internal Medicine is an Open Access article distributed under the Creative Commons Attribution-NonCommercial-NoDerivatives 4.0 International License. To view the details of this license, please visit (https://creativecommons.org/licenses/ by-nc-nd/4.0/).

(C) 2017 The Japanese Society of Internal Medicine

Intern Med 56: 2871-2878, 2017 